\title{
ANALYSIS OF POSSIBILITIES OF INCREASING THE SPANNED DISTANCE USING EDFA AND DRA IN DWDM SYSTEM
}

The aim of this article is a comparison of the EDFA (Erbium Doped Fibre Amplifier) and the DRA (Distributed Raman Amplifier) in a fully optical communication system, working on a specific wavelength. The amplifiers are compared based on their position in the optical loop and are evaluated according to the Q-factor and bit error rate (BER). In the case of the DRA, the accomplished distance was smaller, while changing the optical power in CW (Continuous Wave) to attain better BER. In the application of the EDFA a similar optical loop was created, as well, while the basic parameter for comparison was a bit speed. For the EDFA the bit speed was changed from 10 to 15 Gbps. For the DRA the change was in the power from 1 to $3 \mathrm{~mW}$ with the bit speed kept at $10 \mathrm{Gbps}$. This publication also focuses on the need for such application of a transfer system in a software environment. It would enable examination and research and consequently a minimising of the influence of non-linear effects impacting the particular WDM (Wavelength Division Multiplex) system.

Keywords: BER, DRA, EDFA, WDM.

\section{Introduction}

Our age cannot be called anything but the age of information and telecommunication technologies. Year by year a heightened demand for information is recorded, enabling new services, especially in the area of telecommunication. The internet connection is a matter of course for every household. With it is also connected a greater demand for services like video on demand (VoD), video or music streaming, VoIP and many others. The growing number of users who need to transfer a still greater amount of data though the current infrastructure leads to many problems which the current data networks find hard to manage [1 - 3]. One of these problems is the bandwidth. Until recently the most widespread type of medium for network transfer was metallic wiring. However they reached their physical limits of capacity and bandwidth. Metallic wiring is becoming more and more insufficient. Because of that there had to be found a new transfer medium. This new type of medium is optical fibre.

One convenient faculty of optical fibre springs from the property of light. It is a large bandwidth limited only by the frequency of the light mode. Optical fibres are preferred mostly due to their ability to ensure a faster transfer and of a greater quality for a large amount of data in a very short time. Nowadays optical fibre enables the transfer of data in amounts up to 10
Gbps - 40 Gbps [4 - 6]. The capacity of optical networks can be additionally enlarged by using the technology of the WDM. The WDM enables several channels to be transferred while using one physical optical fibre. Thanks to the WDM systems it is possible to attain the transfer speed of 1 Tbps. The increasing number of transfer speeds for ever-lengthening distances is also beginning to reach the limits for optical fibre. The main factor influencing the quality and reliability is the attenuation and the dispersion of the optical fibre [7, 8]. In addtion, one cannot omit mentioning such non-linear effects as, for example: SPM (Self Phase Modulation), XPM (Cross Phase Modulation) and FWM (Four Wave Mixing), which significantly influence the signal transfer by the optical fibres [9].

\section{Basic theory about the EDFA and the Raman amplifier}

At the signal transfer by the optical communication system on long distances $(>100 \mathrm{~km})$ there can be an attenuation of the transferred signal. This loss is necessary to compensate somehow. The most sensible way of compensation in this case is using amplifiers. The optical amplifiers are very convenient mostly when it is necessary to cross great distances between the optical transmitter and the receiver. In the past the electronic

\footnotetext{
* ${ }^{1}$ Tomas Ivaniga, ${ }^{2}$ Petr Ivaniga, ${ }^{1}$ Jan Turan, ${ }^{1}$ Lubos Ovsenik

${ }^{1}$ Department of Electronics and Multimedia Communications, Faculty of Electrical Engineering and Informatics,

University of Technology Kosice, Slovakia

${ }^{2}$ Department of Information Networks, Faculty of Management Science and Informatics, University of Zilina, Zilina, Slovakia

E-mail: tomas.ivaniga@tuke.sk
} 
regeneration systems were used which performed the opto-electric conversion, to increase the level of signal in the electrical area and finally to perform the electrical-optical conversion [9]. With the entrance of the optical amplifiers these conversions are no longer needed.

Optical amplifiers are used in one-cable and multi-cable optical communication systems. The optical amplifier is a component of the optical network which can markedly influence the quality of the transferred signal. The placement of the optical amplifier within the optical network and its particular amplification greatly influences the occurrence of the nonlinear effects, for example: SPM, XPM and FWM.

\subsection{Erbium Doped Fibre Amplifier}

The history of the EDFA dates back to the 60 's in the $20^{\text {th }}$ century. However the real application of the EDFA started in the 90's. The discovery of this technology is an important milestone in the development of the optical communication systems. Since then the EDFA is used in a wide range of applications wideband optical amplifiers, optical sources or tuneable lasers, and it can be found also in the optical coherent tomograph. The EDFA is successfully used mostly in the WDM transfer systems. The EDFA enabled the constructors of optical communication systems to use an optical window around $1.55 \mu \mathrm{m}$ for transfers $[9,10]$. It also significantly contributed to the increase of the transfer speed and the transfer distance. Erbium-doped fibre is a type of silicate optical fibre with its nucleus doped by ions of Erbium $\mathrm{Er}^{3+}$. The electrons of the erbium-doped optical fibre can be excited into the higher energy levels by the excitation of light with a shorter wavelength. The EDFA is very effective in bands $\mathrm{C}(1530 \mathrm{~nm}-1560 \mathrm{~nm})$ and $\mathrm{L}(1570 \mathrm{~nm}-1610 \mathrm{~nm})$ [11 - 13]. The ideal characteristic of gain for the EDFA is reachable within 1530 $\mathrm{nm}-1560 \mathrm{~nm}$. A typical gain of the erbium-doped optical amplifier was 20-30 dB for a distance of approximately 10 metres. Maximal reachable power on the output of the amplifier is limited by the power of the pumped light source. The value of this power can be between 20-50 $\mathrm{mW}$ (13-17 dBm).

The important factor significantly influencing the effectivity of the EDFA is the choice of the pumped light source. In practice are used light sources pumping light of wavelength of $980 \mathrm{~nm}$ and $1480 \mathrm{~nm}$. The use of the pumping light source of the wavelength of $980 \mathrm{~nm}$ leads to high values of amplification and relatively low values of noise. The usage of wavelength of $1480 \mathrm{~nm}$ for the pumping light source produces larger values of amplification than when using the wavelength of $980 \mathrm{~nm}$, but this light source causes a lower effectivity and a higher noise. In practice is common to use also both types of the pumping sources $[14,15]$. On the first level the pumping light source of $980 \mathrm{~nm}$ ensures the low noise and a sufficient gain. A high output power is attained in the second level by using the pumping light source with wavelength of $1480 \mathrm{~nm}$ [16]. With such ways we can reach low noise and high output power of the EDFA. A typical course of gain and saturated output power are on the Fig. 1.

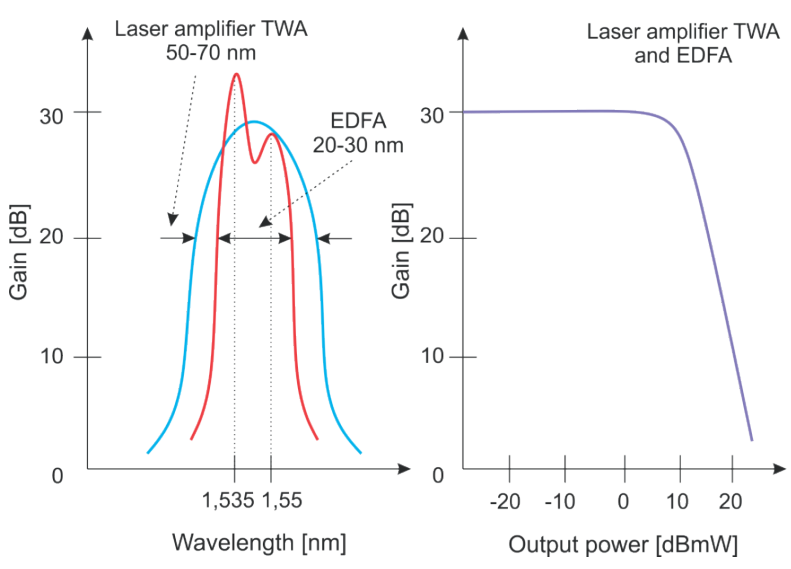

Fig. 1 Characteristics of the EDFA: a) typical gain profile b) typical saturated output power

\subsection{Raman amplifier}

For the purpose of intensifying the optical signal the Raman amplifier can also be used. Basically it is a laser source of radiation connected to the optical line. Signal strengthening utilises the Raman scatter on particles of the waveguide material. The energy transfer from lower wavelengths (wavelength of the radiation of the Raman pump) to higher (wavelengths of the transferred signal) also happens during the scatter [16]. Based on that the signal gains intensity right in its own fibre of the transferred line. This type of amplifier does not reach the values of the EDFA, the level of signal can be amplified by 15 to $20 \mathrm{~dB}$. Raman amplifier is placed at the end of the transfer optical fibre and the radiation of the laser pump flows against the intensified signal [17]. It can be used to strengthen any wavelength under the condition of a correct choice of the wavelength of the laser source. EDFA and Raman amplifier can be conveniently and mutually combined [18]. However these amplifiers also intensify the distortion and so with long distances there has to be another classic repeater connected due to the signal recovery.

\subsubsection{Gain of Raman amplifier}

The wavelength of generated optical radiation is shifted up to $30 \mathrm{THz}$ compared to the original wavelength. Maximal gain ( $30 \mathrm{~dB}$ ) can be observed at the shift by $13 \mathrm{THz}$. For the practice it translates as the necessity to use pumping source working on wavelength of $1450 \mathrm{~nm}$ for the amplifier working on the wavelength of $1550 \mathrm{~nm}$. The power of the amplifier depends on the power and wavelength of the pump, spectral effectivity, the 
length of the fibre and on the size of the mode surface. The most convenient medium to generate optical gain are fibres with small effective mode surface of the nucleus of the fibre $[17,18]$. The Fig. 2 portrays the profile of the gain of the Raman amplifier. The non-linear fibres seem optimal and the fibres for suppression dispersion. In practice it is used the combination of a singlemode fibre with the step change of the refractive index SMF (Single Mode Fibre), fibres compensating the dispersion DCF (Dispersion Compensating Fibre), or also fibres with the shifted dispersion DSF (Dispersion Shifted Fibre).

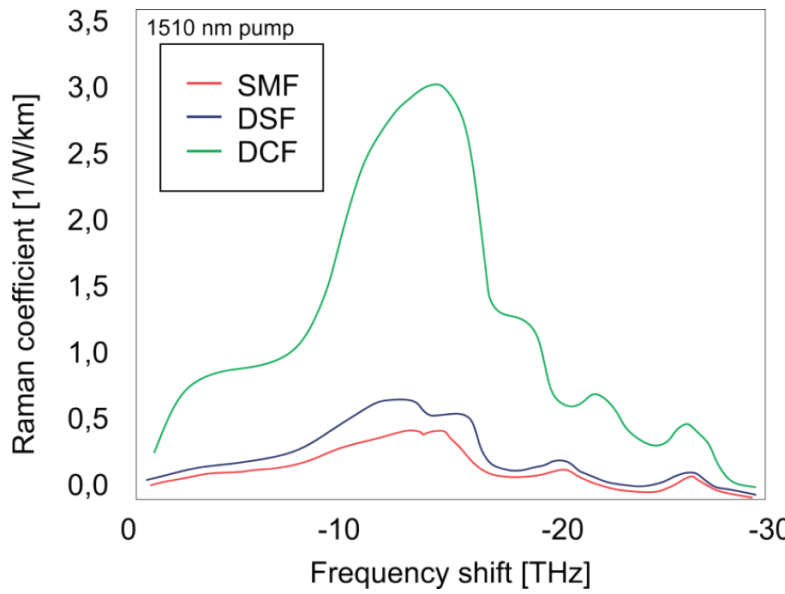

Fig. 2 Gain profile of the Raman amplifier

\subsubsection{Pumping sources for the Raman amplifier}

The source of the Raman amplifier is optical radiation. The pump radiation has shorter wavelength than the intensified optical signal. The contra-flow pumping is more beneficial because of lower noise and limitation of the gain ripple (the whole line absorbs generated influence of the spontaneous emission ASE) [19 - 21]. In addtion a greater number of pumping sources can be used, for example at the beginning or the end of the line - mostly for the maximal gain of the amplifier functioning as the booster. Regimes of the amplifiers' work depend on the placement of the pump [22]. There are two regimes of work:

- DRA (Distributed Raman Amplifier)-with expanded amplifier. This regime requires contra-flow pump. If the optical pumping is placed at the opposite end of the line, the whole line contributes to the gain. These amplifiers are notable for their lower noise, greater gain and lesser non-linear distortion. The disadvantage is the limitation of the maximal number of working wavelengths. In practice it is necessary to connect more amplifiers into the transfer band.

- LRA (Lumped Raman Amplifier)-discrete regime. The amplifier creates one block placed at a certain part of the line. It enables the gain generation at certain wavelengths - usually the ones not available for the amplifiers utilising doped mixtures of rare elements. The LRA can work for example in telecommunication band $\mathrm{S}$ where it enables to attain twice the transfer capacity than EDFA in C-band.

\section{Published results}

Ivaniga, et al., 2016 [23], designed a two-channel WDM system to examine the SPM effect. The aim of this article is to point to the non-linear effect SPM present in fully optical communication systems. Based on that article individual powers of $\mathrm{CW}$ and also the EDFA placement in the optical communication system were set. Because DWDM was created according to ITU-T G.694.1 it was necessary to prevent FWM. The individual simulations started at $12.5 \mathrm{GHz}, 25 \mathrm{GHz}$ and then $50 \mathrm{GHz}$ with gaps between the channels. At $50 \mathrm{GHz}$ FWM did not express in such prominent way as in the UDWDM system [23]. Ivaniga, et al., 2017 [24], formed a four-channel DWDM where they compared the EDFA and the SOA in band C. Individual amplifiers were placed as IN-Line amplifiers and were compared according to BER for two wavelengths. These amplifiers were not compared in order to reach the maximal distance but to compare it in two specific wavelengths. With that contribution it was possible to choose the length of the erbium-doped fibre as $10 \mathrm{~m}$ [24]. Bobrovs, et al., 2013 [25], designed DWDM where they compared Raman-SOA and Raman-EDFA in sixteen-channel DWDM system. Their article also summarised the individual comparisons and the advantages of given connections. Based on the stated distances there were created optical loops in our system [25]. Olonkins, et al., 2016 [26], presented the comparison of EDFA and LRA for eight-channel DWDM system at the speed of $10 \mathrm{Gbps}$ with the modulation of type NRZ. In their simulations the placement of the amplifier was as that of the pre-amplifier. Based on those results we have set NRZ modulation in the receiving part [26]. Nain et al., 2016 [27], examined the non-linear effects in the WDM system. Thanks to this article it was possible to set the length of fibre in every loop. The authors focused on the improvement or the decrease of BER, Q-factor at the change of dispersion and the optical fibre [27]. Ivaniga, et al., 2017 [28], designed a single-channel optical line were the necessity of optical amplifiers after certain distance was proven. The length of fibres and the parameter of amplification were conducted based on those values. In addtion it includes basic mathematical process of calculating BER and Q-factor [28]. Because of the attained results and the publicised articles we have designed our own system for the EDFA and the DRA comparison. 


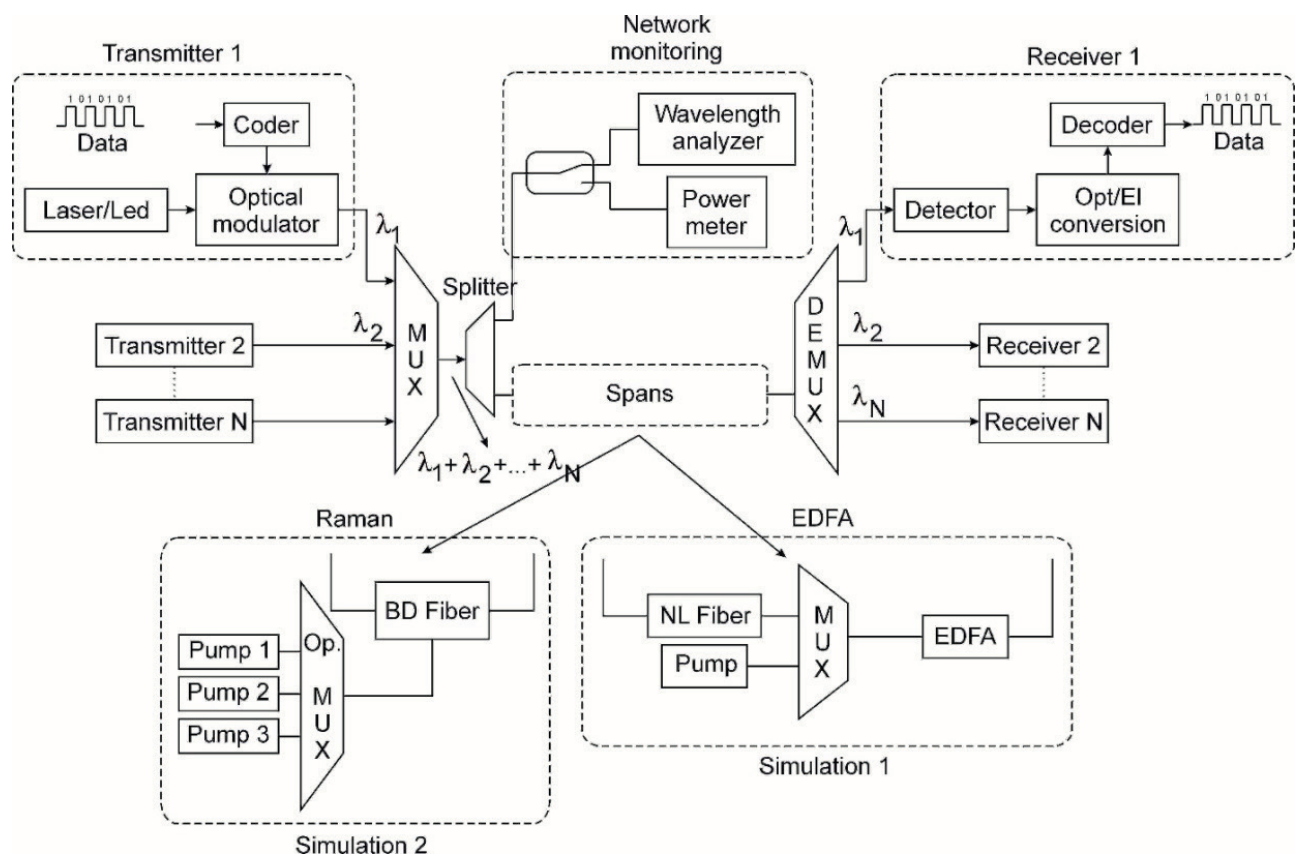

Fig. 3 Topology of the connection for the comparison between EDFA and DRA

\section{Simulation and setting the parameters for EDFA and DRA}

This section contains comparison of the EDFA and the DRA in optical span. These amplifiers were chosen for purpose of increasing maximal distance of communication path. The whole optical communication system contains three elementary parts: transmitting, receiving and transfer part (Fig. 3). There were two simulations carried out, for each one was used a unique transfer part. This was necessary to do for the comparison of BER for the individual systems.

\subsection{Transmitting part}

The transmitting part is created by four basic blocks. The first block is the source of data, able to generate various types of binary sequences. In our case it is set as the block PRBS (Pseudo - Random Binary Sequence) for every transfer channel set to produce the maximal length of the pseudo-random binary sequences. PL (Pattern Length) is exactly 7 (the amount of bits generated in the bit sequence). The number of zero bits at the beginning of the sequence equals 2 and at the end of the sequence 3. The bit speed is set to $10 \mathrm{Gbps}$ during all simulations of the DRA and with the EDFA it increases to $15 \mathrm{Gbps}$. The function of the modulator is to ensure the conversion of the input binary signal into the output electrical signal. The electrical signal is generated by the On - off - ramp (electrical signal is generated by the period of rise and decay periods and jitter's timing). The used modulation is of the type NRZ (Non Return to Zero), the output value is expressed in Volt units with the amount of units per bit in the electrical signal has the value of 5 . The maximal value of the output electrical signal $V_{\max }$ is 1 and the minimal value of the output signal $V_{\min }$ is 0 . This block contains two types of ports: the first is logical with the input values from PRBS and the second is electric with the output values from the generator. The next created block is the Laser. Designed by us the $\mathrm{CW}$ laser has these defined parameters: power, RIN (Relative Intensive Noise), wavelength, phase and linewidth. The transmitted power in every block is established on the level $1 \mathrm{~mW}$ and RIN has the value of $-150 \mathrm{~dB} / \mathrm{Hz}$. In case of the DRA the power with increment $1 \mathrm{~mW}$ the changed to manage a greater distance. The last block is the modulator. We chose the model of an electro-optical modulator using the modulation of the type MZ (Mach Zhender). Given signal then travels into the multiplexor where the optical signals are joined. Our created system has 8 channels with the gap of $50 \mathrm{GHz}$ according to the ITU-T G.694.1, which is basically a standard DWDM. In our simulations we follow only one channel with the wavelength of $1550 \mathrm{~nm}$ to span the distance with a sufficient BER.

\subsection{Transfer part}

The whole transfer part in both topologies is unique. For the EDFA the emphasis is on spanning the distance and it incorporates a different bit speed. For the DRA the distance used was smaller but the change was in the input power of the $\mathrm{CW}$ laser. 


\subsubsection{Optical loop connected to the EDFA}

In the Configuration connected to EDFA these following components are in an optical loop: optical fibre, multiplexor, laser pump and EDFA (Fig. 3). In order to span the greatest possible distance the number of iterations changes in the loop. The optical fibre is set to a constant distance of $75 \mathrm{~km}$. This fibre represented the attenuation of $0.33 \mathrm{~dB} / \mathrm{km}$ during all simulations. The dispersion $\lambda_{0}$ was set to the value of $1,312 \mu \mathrm{m}$ and the group index $n_{1}$ to the value of 1.4786 . The chosen non-linear model was constant, representing non-linear refractive index of $n_{2} 2.6 .10^{-20}$, the diameter was $8.2 \mu \mathrm{m}$. During the simulations the PMD, SBS or Raman effect were ignored. This constructed model represented the input optical and also the output optical port. After the fibre is the multiplexor. The multiplexor contains two input optical ports and one output optical port. Into the first port the signal enters from the optical fibre and into the second enters the signal from the laser pump. Our designed model has the wavelength set to $980 \mathrm{~nm}$ with the power of $4.39 \mathrm{~mW}$. RIN equalled $-150 \mathrm{~dB} / \mathrm{Hz}$ and timestpas was set to 3.125 ps. The key component in this loop is the EDFA. On Fig. 4 is the line of gain/loss with the wavelength for our designed model EDFA. The EDFA has the saturation parameter set to the value of $3.10^{15} \mathrm{~m}^{-1} \mathrm{~s}^{-1}$

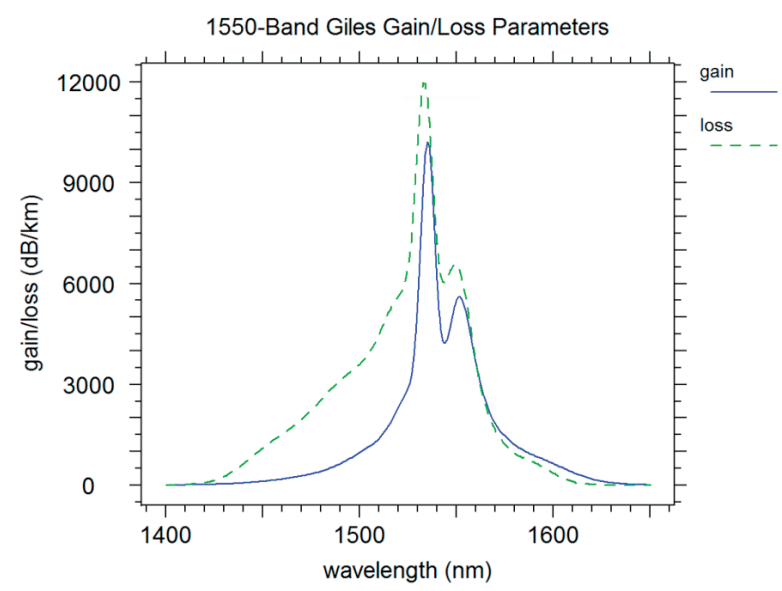

Fig. 4 Band Giles Gain/Loss Parameters

The aim of the simulation was the formation of a loop. One loop represented the trajectory of the optical signal through the fibre of $75 \mathrm{~km}$ and its following amplification. This loop increases with the increment 5 . This means that the loop with the number of iterations 10 passes through the amplifier 10 times and the distance is $750 \mathrm{~km}$.

\subsubsection{Optical loop connected to the DRA}

When the DRA was connected to the optical loop, there are three laser pumps used, an optical multiplex and a Bi-directional non-linear optical fibre. The first laser pump had the power set to $118 \mathrm{~mW}$ at the wavelength of $1450 \mathrm{~nm}$ and RIN for every pump equals $-150 \mathrm{~dB} / \mathrm{Hz}$. The second laser pump has the value of 82 $\mathrm{mW}$ and it worked at the wavelength of $1458 \mathrm{~nm}$. The third laser pump had $81 \mathrm{~mW}$ and was using the wavelength of $1447 \mathrm{~nm}$. Signals enter the optical multiplex were they are joined and they proceed into the fibre. On Fig. 5 are the lines for the three laser pumps with the change of power and distance.

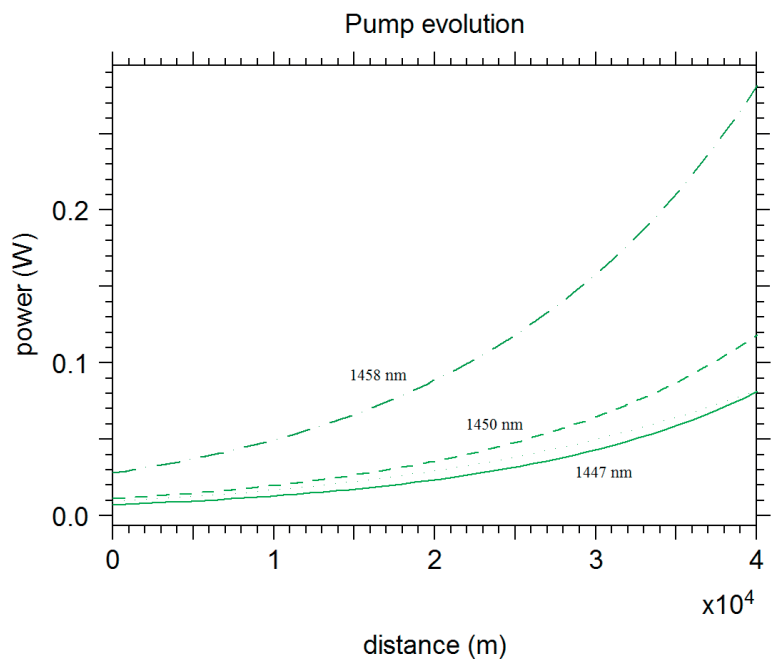

Fig. 5 Pump evolution for 3 laser pumps

The created model of the optical fibre represents a smaller distance, only a $50 \mathrm{~km}$. The total attenuation was $0.3 \mathrm{~dB} / \mathrm{km}$. The dispersion $\lambda_{0}$ was set to the value of $1,312 \mu \mathrm{m}$ and the group index $n_{1}$ to the value of 1.4786 . The chosen non-linear model was constant which meant the non-linear refractory index $n_{2} 2.6 .10^{-20}$, the diameter was $8.2 \mu \mathrm{m}$. In addtion in this case the PMD and SBS were turned off but the Raman effect was turned on here. The Raman gain for the particular wavelength was $9.8 .10^{-14}$ $\mathrm{m} / \mathrm{W}$ and the wavelength was $1 \mu \mathrm{m}$. The setting of the Rayleigh scattering coefficients for $k$ equals $2.16 .10^{-7} \mathrm{~m}^{-1}$ and $m$ equals 2.7 with the set temperature of $298.15 \mathrm{~K}$.

\subsection{Receiving part}

The receiving part of the fully optical communication system is the same in both topologies. The aim of this part is the evaluation of quality of the optical line based on BER and also the related Q-factor. The representation of the simulated values is according to the MC method (Monte Carlo). The filter in the receiver is the Bessel type and the quantum effectivity is 0.8 . The component is designed to receive the input optical signal and at the output the signal is electrical. In Figs. 6 and 7 are the measured values of BER. In Fig. 6 are the acceptable values for EDFA with $2625 \mathrm{~km}, 10 \mathrm{Gbps}$ and BER equals 6.4325.10-11. 
In Fig. 7 are the acceptable values for DRA $700 \mathrm{~km}, 10 \mathrm{Gbps}$ and BER equals 7.1877.10-7 while the power in this case was $3 \mathrm{~mW}$. The measured values after connecting EDFA with the changed bit speed are in Table 1. The measured values for the DRA with changed power are in Table 2.

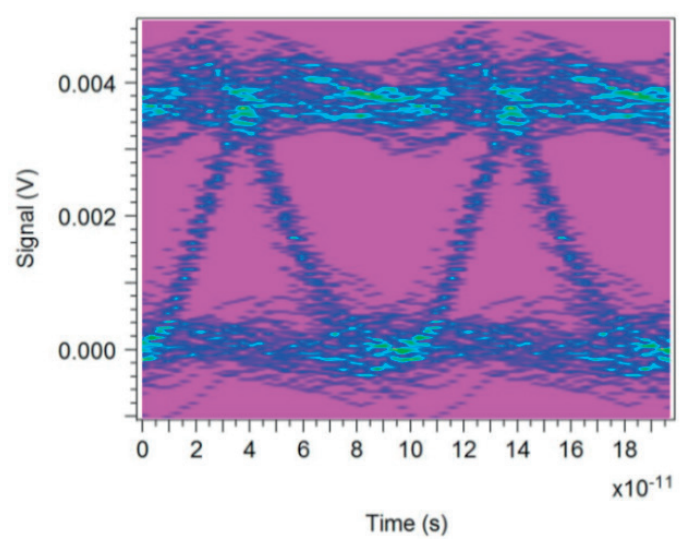

Fig. 6 BER for the EDFA with the distance of $2625 \mathrm{~km}$
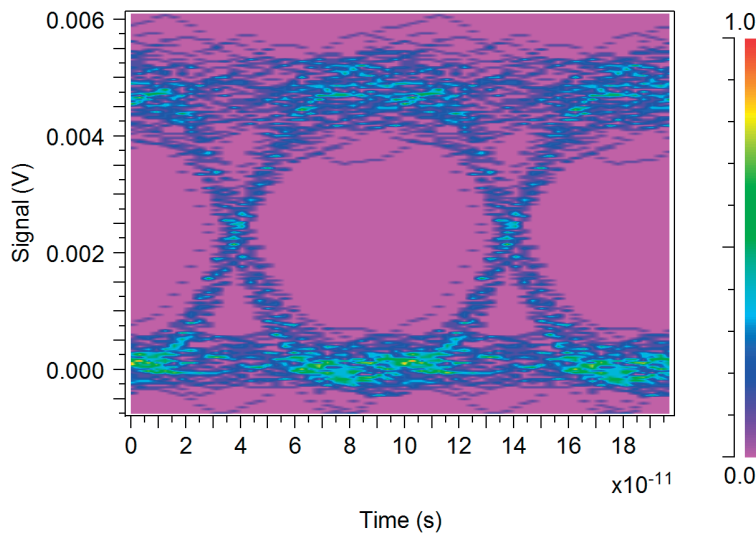

Fig. 7 BER for the DRA at length $700 \mathrm{~km}$ and power of $3 \mathrm{~mW}$

Simulated values for the EDFA configuration

Table1

\begin{tabular}{ccccc}
\hline $\begin{array}{c}\text { Distance } \\
{[\mathrm{km}]}\end{array}$ & $\begin{array}{c}\text { BER/ } \\
\mathbf{1 0} \mathrm{Gb} p s\end{array}$ & $\begin{array}{c}\text { Q-factor/ } \\
\mathbf{1 0} \mathbf{G} b p s\end{array}$ & $\begin{array}{c}\text { BER/ } \\
\mathbf{1 5} \mathbf{~ G b p s}\end{array}$ & $\begin{array}{c}\text { Q-factor/ } \\
\mathbf{1 5} \mathbf{~ G b p s}\end{array}$ \\
\hline 1500 & $6.9258 .10^{-28}$ & 10.883 & $3.7513 .10^{-13}$ & 7.1699 \\
1875 & $1.6492 .10^{-20}$ & 9.2088 & $5.9885 .10^{-10}$ & 6.0806 \\
2250 & $1.4174 .10^{-14}$ & 7.6057 & $5.8837 .10^{-7}$ & 4.8595 \\
2625 & $6.4325 .10^{-11}$ & 6.4288 & $7.2964 .10^{-5}$ & 3.7979 \\
3000 & $1.5673 .10^{-7}$ & 5.1152 & $3.1125 .10^{-3}$ & 2.9987 \\
3150 & $2.2292 .10^{-6}$ & 4.5888 & 0 & 0 \\
\hline
\end{tabular}

Simulated values with the DRA connected

Table 2

\begin{tabular}{cccc}
\hline Distance $[\mathrm{km}]$ & Power[mW] & BER & Q-factor \\
\hline 500 & 1 & $1.9696 .10^{-23}$ & 9.9055 \\
550 & 1 & $2.6630 .10^{-13}$ & 7.2167 \\
600 & 1 & $1.0001 .10^{-7}$ & 5.1993 \\
650 & 1 & $9.8651 .10^{-5}$ & 3.7224 \\
700 & 1 & $4.4423 .10^{-3}$ & 2.6165 \\
700 & 2 & $7.1877 .10^{-7}$ & 4.8197 \\
700 & 3 & $7.0922 .10^{-12}$ & 6.7560 \\
750 & 2 & $2.8737 .10^{-4}$ & 3.4433 \\
750 & 3 & $5.8286 .10^{-7}$ & 4.8614 \\
750 & 3 & $2.5457 .10^{-4}$ & 3.4759 \\
\hline
\end{tabular}

\section{Conclusion}

The aim of this article was a comparison of two optical fibre amplifiers, namely the EDFA and the DRA. In the configuration with EDFA the distance was $2625 \mathrm{~km}$ at the bit speed of $10 \mathrm{Gbps}$, while BER reached the value of $6.4325 .10^{-11}$ (Fig. 6). An optical line is considered as acceptable when the bit error rate is under $10^{-12}$. Changing the bit speed to $15 \mathrm{Gbps}$ the distance becomes $1500 \mathrm{~km}$ with BER of $3.7513 .10^{-13}$. With the configuration with DRA the distance was $700 \mathrm{~km}$ with the power of $3 \mathrm{~mW}$ which corresponded with BER of $7.0922 .10^{-12}$. The designed system was constructed to compare the distance coverage from the placement viewpoint. When choosing the correct setting it is necessary to consider non-linear effects, the distance, price or the number of users. The choice will be influenced by the compromise of whether the provider decides to lower the price (which decreases the bit speed) or increases the bit speed and shorten the distance of the optical line, or whether it decides to use more frequent channels where the FWM can interfere, etc. That is why the analysis of the individual optical networks in simulation programmes is an essential part of the design of all new optical communication networks. After an appropriate number of simulations are provided to address all of the customers' demands it is possible to create the most effective model taking into consideration all undesirable effects influencing the particular optical network.

\section{Acknowledgment}

This work was supported by research grant KEGA No. 023TUKE-4/2017. 


\section{References}

[1] SMIESKO, J., URAMOVA, J.: Access Node Dimensioning for IPTV Traffic Using Effective Bandwidth. Communications Scientific Letters of the University of Zilina, 14(2), 11-16, 2012.

[2] STEINGARTNER, W., NOVITZKA, V.: A New Approach to Semantics of Procedures in Categorical Terms. Proc. of IEEE $13^{\text {th }}$ intern. scientific conference on informatics (INFORMATICS 2015), Serbia, 7377842, 252-257, 2015.

[3] RUZBARSKY, J., TURAN, J., OVSENIK, L.: Stimulated Brillouin Scattering in DWDM All Optical Communication Systems. 26 ${ }^{\text {th }}$ intern. conference radioelektronika (RADIOELEKTRONIKA 2016), Slovakia, 7477354, 395-398, 2016.

[4] ABDUllaeV, A., TURAN, J.: Survey of the Problems and Solutions of Arrayed Waveguide Gratings Used in the Optical Network. Acta Electrotechnica et Informatica, 14(3), 49-53, 2014.

[5] MIKUS, L.: Evaluations of the Error Rate in Backbone Networks. Elektrorevue, 12(2), 1-6, 2010.

[6] UDAYAKUMAR, R., KHANAA, V., SARAVANAN, T.: Chromatic Dispersion Compensation in Optical Fiber Communication System and its Simulation. Indian Journal of Science and Technology, 6, 4762-4766, 2013.

[7] SMIESKO, J.: IP Network Management of Sources for IP Traffics (in Slovak). Scientific Papers of the University of Pardubice, Series D: Faculty of Economics and Administration, 21(32), 109-117, 2014.

[8] IVANIGA, T., OVSENIK, L., TURAN, J.: The Four-channel WDM System Using Semiconductor Optical Amplifier. $26^{\text {th }}$ intern. conference radioelektronika (RADIOELEKTRONIKA 2016), Slovakia, 7477350, 354-357, 2016.

[9] SIFTA, R., MUNSTER, P., KRAJSA, O., FILKA, M.: Simulation of Bidirectional Traffic in WDM-PON Networks (in Polish). Przeglad Elektrotechniczny, 90(1), 95-100, 2014.

[10] AZAWE, M. I.: Low Noise C-band EDFA/DRA Hybrid Amplifier Using the Same Pump Laser Diodes. Photonics Letters of Poland, 3(4), 165-167, 2011.

[11] STEINGARTNER, W., NOVITZKA, V.: Categorical Model of Structural Operational Semantics for Imperative Language. Journal of Information and Organizational Sciences, 40(2), 203-219, 2016.

[12] OLONKINS, S., BOBROVS, V., IVANOVS, G.: Investigation of Fiber Optical Parametric Amplifier Performance in DWDM Transmission Systems. Elektronika ir Elektrotechnika, 20(1), 88-91, 2014.

[13] ZYSKIND, J.: Erbium-Doped Fiber: Amplifiers: What Everyone Needs to Know. Optical Fiber Communications Conference and Exhibition (OFC 2016), USA, 7537552, 2016.

[14] SHARMA, S., MOHAN, S.: Countering the Gain Behavior of Erbium Doped Fiber Amplifiers: A Cross Layer Aapproach. IEEE intern. conference on Advanced Networks and Telecommunications Systems (ANTS 2013), India, 6802885, 2013.

[15] LIPTAI, P., MORAVEC, M., LUMNITZER, E., LUKACOVA, K.: Impact Analysis of the Electromagnetic Fields of Transformer Stations Close to Residential Buildings. SGEM, 1, 355-360, 2014.

[16] LUCERO, A.: Advanced Amplifier Schemes in Long-Haul Undersea Systems. Zyskind, J., Srivastava, A. (Eds.), Optically Amplified WDM Networks, Elsevier, USA, 253-276, 2011.

[17] AGALLIU, R., LUCKI, M.: System Performance and Limits of Optical Modulation Formats in Dense Wavelength Division Multiplexing Systems. Elektronika ir Elektrotechnika, 22(2), 123-129, 2016.

[18] OLONKINS, S., STANKUNOVS, I., ALSEVSKA, A., GEGERE, L., BOBROVS, V.: Investigation of In-Line Distributed Raman Amplifiers with Co and Counter-Propagating Pumping Schemes. Proc. of Progress in Electromagnetics Research Symposium (PIERS 2016), China, 7735423, 3773-3777, 2016.

[19] FELINSKYI, G. S., DYRIV, M. Y.: Noise Gain Features of Fiber Raman Amplifier. Advances in OptoElectronics, 5843636, 2016.

[20] TITHI, F. H., MAJUMDER, S. P.: Performance Limitations Due to Combined Influence of ASE and Raman Amplifier Induced Crosstalk in a WDM System with Direct Detection Receiver. $9^{\text {th }}$ intern. conference on Electrical and Computer Engineering (ICECE 2016), Bangladesh, 566-569, 2016.

[21] AL-KHATEEB, M. A. Z., TAN, M., IQBAL, M. A., MCCARTHY, M., HARPER, P., ELlIS, A. D.: Four Wave Mixing in Distributed Raman Amplified Optical Transmission Systems. IEEE Photonics Conference (IPC 2016), USA, 7831072, 795-796, 2016.

[22] MASUDA, H., TOMIZAWA, M., MIYAMOTO, Y., HAGIMOTO, K.: Impacts of Distributed Raman Amplification Transmission Technologies on Terrestrial Large-Capacity WDM Systems. Electronics and Communications in Japan, part I: Communications (English translation of Denshi Tsushin Gakkai Ronbunshi), 90(6), 20-28, 2007.

[23] IVANIGA, T., OVSENIK, L., TURAN, J.: Investigation of SPM in WDM System with EDFA. Carpathian Journal of Electronic \& Computer Engineering, 9(2), 7-12, 2016.

[24] IVANIGA, T., IVANIGA, P.: Comparison of the Optical Amplifiers EDFA and SOA Based on the BER and Q-factor in C-band. Advances in Optical Technologies, 9053582, 2017. 
[25] BOBROVS, V., OLONKINS, S., ALSEVSKA, A., GEGERE, L., IVANOVS, G.: Comparative Performance of Raman-SOA and Raman-EDFA Hybrid Optical Amplifiers in DWDM Transmission Systems. International Journal of Physical Sciences, 8(39), 18981906, 2013.

[26] OLONKINS, S., BOBROVS, V., PILATS, D., PORINS, J.: Comparison of EDFA and LRA Preamplifier Performance in WDM Transmission Systems. Proc. of Progress in Electromagnetics Research Symposium (PIERS 2016), China, 7735424, 3778-3782, 2016.

[27] NAIN, H., JADON. U., MISHRA. V.: Evaluation and Analysis of Non-Linear Effect in WDM Optical Network. IEEE intern. conference on Recent Trends in Electronics, Information \& Communication Technology (RTEICT 2016), India, 36-39, 2016.

[28] IVANIGA, P., IVANIGA, T.: 10 Gbps Optical Line Using EDFA for Long Distance Lines (in Polish). Przeglad Elektrotechniczny, 93(3), 193-196, 2017. 